\title{
Cardiorespiratory parameters in newborns during sedation with chloral hydrate
}

\author{
Petja Fister ${ }^{1}$, Jerneja Pecek ${ }^{1}$, Barbara Gnidovec Strazisar ${ }^{2}$, Darja Paro-Panjan ${ }^{1 \oplus}$ \\ ${ }^{1}$ Division of Neonatology, Department of Pediatrics, University Medical Centre, Ljubljana; ${ }^{2}$ Department of Pediatrics, General Hospital \\ Celje, Slovenia.
}

\begin{abstract}
We commonly use chloral hydrate sedation in newborns, though its cardiorespiratory side effects have not yet been fully investigated. Our study aimed to analyze the impact of chloral hydrate on cardiorespiratory parameters in term newborns. We performed a prospective, pre-post single-arm interventional study in 42 term, respiratorily and hemodynamically stable newborns. Oxygen saturation $\left(\mathrm{SpO}_{2}\right)$, end-tidal $\mathrm{CO}_{2}\left(\mathrm{ETCO}_{2}\right)$, the apnea-hypopnea index and the respiratory and heart rates were recorded by polygraphy, starting 0.5 - 1 hour before oral administration of chloral hydrate at a dose of $40 \mathrm{mg} / \mathrm{kg}$ and ceasing 4 hours post-administration. After administration of chloral hydrate, the mean basal $\mathrm{SpO}_{2}$ dropped by $2.0 \%$ (from 97.1\% to 95.1\%; $\mathrm{p}<0.001$ ) and the mean basal $\mathrm{ETCO}_{2}$ increased by $3.9 \mathrm{mmHg}$ (25.6 to $\left.29.5 \mathrm{mmHg} ; \mathrm{p}<0.001\right)$. We found a significant decrease in the minimal $\mathrm{SpO}_{2}$ values $(\mathrm{p}<0.001)$ and an increase in the percentage of time spent with $\mathrm{SpO}_{2}$ $<95 \%$ and $<90 \%(p<0.001)$. The mean increase in the estimated apnea-hypopnea index was 3.5 events per hour $(\mathrm{p}<0.001)$. The mean respiratory and heart rates were significantly lower $150 \mathrm{~min}$ after the administration of chloral hydrate when compared with pre-sedation values (51/min and 127/min versus 61/min and 138/min respectively; $\mathrm{p}$ 0.001). A considerable number of patients exhibited changes in cardiorespiratory parameters that differed considerably from the normal ranges. In conclusion, $\mathrm{SpO}_{2}, \mathrm{ETCO}_{2}$, the estimated apnea-hypopnea index and the respiratory and heart rates changed after the administration of chloral hydrate. They remained within normal limits in most newborns, but the inter-individual variability was high in the studied population.
\end{abstract}

Key words: chloral hydrate, newborn, oximetry, respiration, sedative.

Chloral hydrate is frequently used for light sedation of newborns to facilitate painless diagnostic procedures that require the patients to be motionless, such as various radiological and neurophysiological tests. ${ }^{1-5}$ The recommended dose of chloral hydrate for newborns and infants is $25-50 \mathrm{mg} / \mathrm{kg}$. ${ }^{6}$ The sedative effects of chloral hydrate become clinically apparent approximately 20 minutes after oral or rectal administration $^{7-9}$ and last between $90^{7,8}$ and $165^{10}$ minutes, although they may be prolonged, especially in preterm infants. ${ }^{2}$

In some previous pediatric studies, it has been suggested that chloral hydrate sedation in

¿ Darja Paro-Panjan

darja.paro@kclj.si

Received17th December 2017; revised 12th May 2018; accepted 20th July 2018 infants and children is effective and safe, with a relatively low risk of severe respiratory and hemodynamic adverse effects. ${ }^{1,3,4,8,9}$ On the other hand, some have reported severe complications of chloral hydrate sedation in children and even death. ${ }^{11}$ Chloral hydrate may, like many other sedatives, affect respiratory and cardiovascular function and pose a risk of respiratory depression and hypoxia. ${ }^{12-14}$ This is especially important in newborns, who have unstable breathing patterns due to the immature control center, and in whom the elimination of chloral hydrate may be prolonged.6,15 It has been implied that the risk of hemoglobin desaturation during chloral hydrate sedation is relatively high in newborns ${ }^{16}$ and studies have shown that younger age is a risk factor for sedation-related adverse effects. ${ }^{3,17}$ Still, little data exist on the cardiorespiratory side effects of chloral hydrate in newborns. Previous studies have mainly 
included older infants and children ${ }^{3,5,8,9}$, relied on intermittent measuring of vital signs ${ }^{3,4,8,9}$ or were mostly retrospective, focusing only on clinically apparent adverse events. ${ }^{1,4,16}$

To further clarify the impact of chloral hydrate on cardiorespiratory function in term newborns, we analyzed continuously recorded cardiorespiratory parameters, including arterial oxygen saturation $\left(\mathrm{SpO}_{2}\right)$, end-tidal $\mathrm{CO}_{2}$ $\left(\mathrm{ETCO}_{2}\right)$, the apnea-hypopnea index and the respiratory and heart rates, before and after the administration of oral chloral hydrate at a dose of $40 \mathrm{mg} / \mathrm{kg}$.

\section{Material and Methods}

\section{Patients}

A prospective, pre-post intervention study on a single group of patients, where each newborn served as his or her own control, was conducted between May 2015 and June 2016 at the Department of Neonatology, Division of Pediatrics, University Medical Center Ljubljana, Slovenia. Ethical approval was obtained from the National Medical Ethics Committee on May 26, 2015 (number 40/05/15).

The inclusion criteria were: postmenstrual age at least 37 weeks, chronological age less than 4 weeks and the need for sedation with chloral hydrate for auditory brainstem response (ABR) audiometry. We excluded newborns receiving drugs that have known interactions with chloral hydrate (furosemide, phenytoin, flumazenil and amiodarone) and newborns with congenital cyanotic heart disease, liver failure, renal insufficiency and signs of infection or respiratory compromise.

Parents of all newborns signed an informed consent form prior to their enrollment in the study.

\section{Methods}

Polygraphic recordings of cardiorespiratory parameters were started half an hour to one hour prior to the administration of a single dose of oral chloral hydrate of $40 \mathrm{mg} / \mathrm{kg}$. A second dose was never given. Half an hour later, the $\mathrm{ABR}$ test was performed, during which the recording remained uninterrupted and was continued until 4 hours after the administration of chloral hydrate. During the monitoring, newborns were under constant supervision.

Cardiorespiratory parameters were recorded by an Embletta MPR PG, Natus Medical Incorporated polygraphy device. The respiratory effort was measured by respiratory inductance plethysmography (XactTrace Single Use Cut-to-Fit Embla RIP Belts), nasal airflow by nasal thermistor (Embla Breath Sensor Airflow Thermistor Preemie), $\mathrm{SpO}_{2}$ and the heart rate by pulse oximetry (Nonin Xpod External OEM Pulse Oximeter) and ventilation by $\mathrm{ETCO}_{2}$ (Microstream CapnoLine H Infant/ Neonate nasal cannulas and Comdek Portable Color Oxi-Capnography MD-850, calibrated to assure accuracy). The thermistor and nasal cannulas were positioned in a way that they did not obstruct the nares of the newborns, conforming to the manufacturer's instructions. The newborns were breathing room air and there was no supplemental oxygen given. The noninvasive side-stream capnometry through nasal cannulas has previously been proven to be an accurate method for estimating $\mathrm{ETCO}_{2}$ in nonintubated newborns without pulmonary disease. ${ }^{18-20}$

\section{Data processing}

Polygraphic recordings were displayed and analyzed using Embla RemLogic-E Sleep Diagnostic Software version 3.4.1, calibrated for newborns, infants and children.

Cardiorespiratory parameters were determined for every newborn and for each part of the study (before and after the administration of chloral hydrate). The primary outcomes were: 1) basal $\mathrm{SpO}_{2}$ as a marker of oxygenation; and 2) basal $\mathrm{ETCO}_{2}$ as a marker of ventilation. The secondary outcomes included: 1) percentage of time with $\mathrm{SpO}_{2}<95 \%$; 2) percentage of time 
with $\mathrm{SpO}_{2}<90 \%$; 3) minimal $\mathrm{SpO}_{2}$; 4 ) estimated apnea-hypopnea index; 5) respiratory rate; and 6) heart rate. Normal ranges for these cardiorespiratory parameters were defined according to the published centiles for healthy term newborns..$^{21-25}$

The Embla RemLogic-E Software allowed automatic calculation of the basal and minimal $\mathrm{SpO}_{2}$ and the percentage of time spent in a specified $\mathrm{SpO}_{2}$ zone or below a chosen threshold in selected parts of the recordings.

The basal $\mathrm{ETCO}_{2}$ was estimated manually from the capnometry curve by comparing $\mathrm{ETCO}_{2}$ values before and after the administration of chloral hydrate and determining the most accurate basal value for these two periods.

Apneas and hypopneas were scored manually, according to the American Academy of Sleep Medicine criteria. ${ }^{26}$ We also categorized apneas as central, obstructive or mixed. The apneahypopnea index was defined as the number of apneas and hypopneas per hour of estimated total sleep time. Total sleep time was estimated by manually excluding all parts of the recordings with motion artifacts on account of arousals, movement or handling of newborns.

The respiratory and heart rates were counted in the period before the administration of chloral hydrate and then at 30-minute intervals during sedation. The average respiratory rate over the 30-minute period was counted by analyzing the breathing waveform, derived from the signal from the respiratory inductance plethysmography: we counted the number of breaths in 60 seconds, when the breathing was calm and the respiratory pattern stable. The mean heart rate was calculated automatically by the Embla RemLogic-E Software.

\section{Statistical analysis}

All calculations were performed using Microsoft Excel 2013 and IBM SPSS Statistics version 21. Data are presented either as the mean and standard deviation or the median and interquartile range, depending on the normality of the distribution of observed variables.

For all of the observed variables, the difference between the value before and after the administration of chloral hydrate, as well as the relative change from the baseline pre-sedation value, were calculated for each individual patient. The differences were statistically analyzed using the two-tailed Student's t-test for paired data and the two-tailed Wilcoxon signedrank test. The level of significance (alpha) of 0.05 was used. Because of multiple comparisons, this significance level was adjusted using the Bonferroni correction. Hence, each individual hypothesis was tested at $\alpha=0.006$. Confidence intervals were adjusted accordingly.

\section{Results}

Out of 49 newborns enrolled in the study, seven newborns were excluded due to the poor quality of the recordings. Measurements of $\mathrm{ETCO}_{2}$ were available for 27 newborns. The characteristics of the study population are presented in Table I. Adequate depth of sedation to perform the ABR testing was achieved in all newborns. After we stopped the recording of cardiorespiratory parameters, newborns were awake and back to their baseline alertness.

In most newborns, basal $\mathrm{SpO}_{2}$ was lower and basal $\mathrm{ETCO}_{2}$ was higher after the administration of chloral hydrate compared with pre-sedation values (Table II). The mean basal $\mathrm{SpO}_{2}$ level dropped by $2.0 \%$ (from $97.1 \%$ to $95.1 \%$ ) and the mean basal $\mathrm{ETCO}_{2}$ increased by $3.9 \mathrm{mmHg}$ (from 25.6 to $29.5 \mathrm{mmHg}$ ). The maximum decrease in basal oxygen saturation was $5 \%$. The maximal $\mathrm{ETCO}_{2}$, recorded during sedation, was $38 \mathrm{mmHg}$. We observed a $>20 \%$ increase in $\mathrm{ETCO}_{2}$ in 9 of 27 newborns (33\%).

The decrease in oxygenation was additionally manifested by a higher proportion of time spent at lower $\mathrm{SpO}_{2}$ levels and a decrease in minimal $\mathrm{SpO}_{2}$ during sedation with chloral hydrate.

We also observed an increase in the estimated apnea-hypopnea index. Of all the recorded 
Table I. Baseline characteristics of participating newborns (n: 42).

\begin{tabular}{lc}
\hline Patient Characteristics & Results \\
\hline Male sex, $\mathrm{n}(\%)$ & $28(66.7 \%)$ \\
Age at the time of the study, days & $16 \pm 16$ \\
Gestational age, weeks & $38 \pm 3$ \\
Postmenstrual age at the time of the study, weeks & $40 \pm 2$ \\
Birth weight, gr & $3,107 \pm 803$ \\
Weight at the time of the study, gr & $3,350 \pm 605$ \\
Head circumference at birth, cm & $34 \pm 3$ \\
Head circumference at the time of the study, cm & $35 \pm 1$ \\
Apgar score at 5 min, & $8 \pm 3$ \\
Apgar score of $>8$ at 5 min, $\mathrm{n}(\%)$ & $22(52.4 \%)$ \\
\hline
\end{tabular}

Table II. Primary outcome measures.

\begin{tabular}{lcccc}
\hline Variable & Before Sedation $^{\mathrm{a}}$ & During Sedation $^{\mathrm{a}, \mathrm{b}}$ & $\mathrm{P}^{\mathrm{c}}$ & Difference (99.4\% CI) \\
\hline${\text { Basal } \mathrm{SpO}_{2}, \%}_{\text {Basal } \mathrm{ETCO}_{2}, \mathrm{mmHg}}^{97.1 \pm 1.9}$ & $25.6 \pm 3.3$ & $95.1 \pm 2.4$ & $<0.001$ & -2.0 (-2.7 to -1.4) \\
\hline
\end{tabular}

${ }^{\mathrm{a}}$ Mean \pm SD. ${ }^{\mathrm{b}}$ In the 4-hour period after administration of chloral hydrate. ${ }^{\mathrm{c} T w o}$-tailed Student's t-test for paired data.

ETCO2: end-tidal CO2, SpO2: arterial oxygen saturation.

Table III. Secondary outcome measures.

\begin{tabular}{|c|c|c|c|c|}
\hline Variable & Before Sedation $^{a}$ & During Sedation ${ }^{\mathrm{a}, \mathrm{b}}$ & $\mathrm{Pc}$ & Difference $(99.4 \% \mathrm{CI})^{\mathrm{d}}$ \\
\hline Time with $\mathrm{SpO}_{2}<95 \%, \%$ & $11.0(0.8$ to 26.9$)$ & $30.1(12.4$ to 55.0$)$ & $<0.001$ & 19.1 (11.6 to 26.6$)$ \\
\hline Time with $\mathrm{SpO}_{2}<90 \%, \%$ & $0.0(0.0$ to 0.7$)$ & $1.9(0.6$ to 7.8$)$ & $<0.001$ & Not applicable \\
\hline Minimal $\mathrm{SpO}_{2}, \%$ & $90.0(87.5$ to 93.0$)$ & $83.0(81.0$ to 85.0$)$ & $<0.001$ & Not applicable \\
\hline Apnea-hypopnea index, $\mathrm{n} / \mathrm{h}$ & $0.0(0.0$ to 2.1$)$ & $3.4(0.8$ to 6.9$)$ & $<0.001$ & $3.5(1.7$ to 5.2$)$ \\
\hline Respiratory rate, $\min ^{-1}$ & $60.9 \pm 14.0$ & $51.3 \pm 11.1^{\mathrm{e}}$ & $<0.001$ & $-9.6(-16.7$ to -2.5$)$ \\
\hline Heart rate, $\min ^{-1}$ & $138.1 \pm 17.0$ & $126.8 \pm 13.0^{\mathrm{f}}$ & $<0.001$ & $-11.3(-18.0$ to -4.6$)$ \\
\hline
\end{tabular}

${ }^{a}$ Median $(\mathrm{IQR})$ or mean $\pm \mathrm{SD}$. ${ }^{\mathrm{b} I n}$ the 4 -hour period after administration of chloral hydrate. ${ }^{\mathrm{T}}$ Two-tailed Student's t-test for paired data or two-tailed Wilcoxon signed-rank test. ${ }^{\mathrm{d} C}$ Calculated when data were normally distributed. ${ }^{\mathrm{e}}$ At 150 minutes after administration of chloral hydrate, when the mean respiratory rate was the lowest. ${ }^{\mathrm{f}}$ At 150 minutes after administration of chloral hydrate, when the mean heart rate was the lowest. $\mathrm{SpO}_{2}$ : arterial oxygen saturation.

apneas, $89 \%$ were central in origin, 3\% were obstructive, $1 \%$ were mixed and in $7 \%$, the type of apnea could not be reliably determined. Lastly, chloral hydrate sedation affected the respiratory and heart rates (Table III and Figs 1 and 2).

The mean respiratory rate and the mean heart rate gradually decreased during the course of sedation and were at their lowest 150 minutes after the administration of chloral hydrate (Fig. 2). The mean maximum decrease in the respiratory rate was $10 \pm 15$ breaths per minute and the mean maximum decrease in the heart rate was $11 \pm 14$ beats per minute (Table III). A $>20 \%$ decrease in the respiratory rate was observed in 13 newborns (34\%) and a $>20 \%$ decrease in the heart rate was seen in five newborns (13\%). Of these, one newborn (3\%) exhibited an abnormally low respiratory rate, while an abnormally low heart rate was measured in three newborns (8\%), according to the normative data (lower 10th percentiles for respiratory and heart rates in healthy term newborns)..$^{21,22}$ 


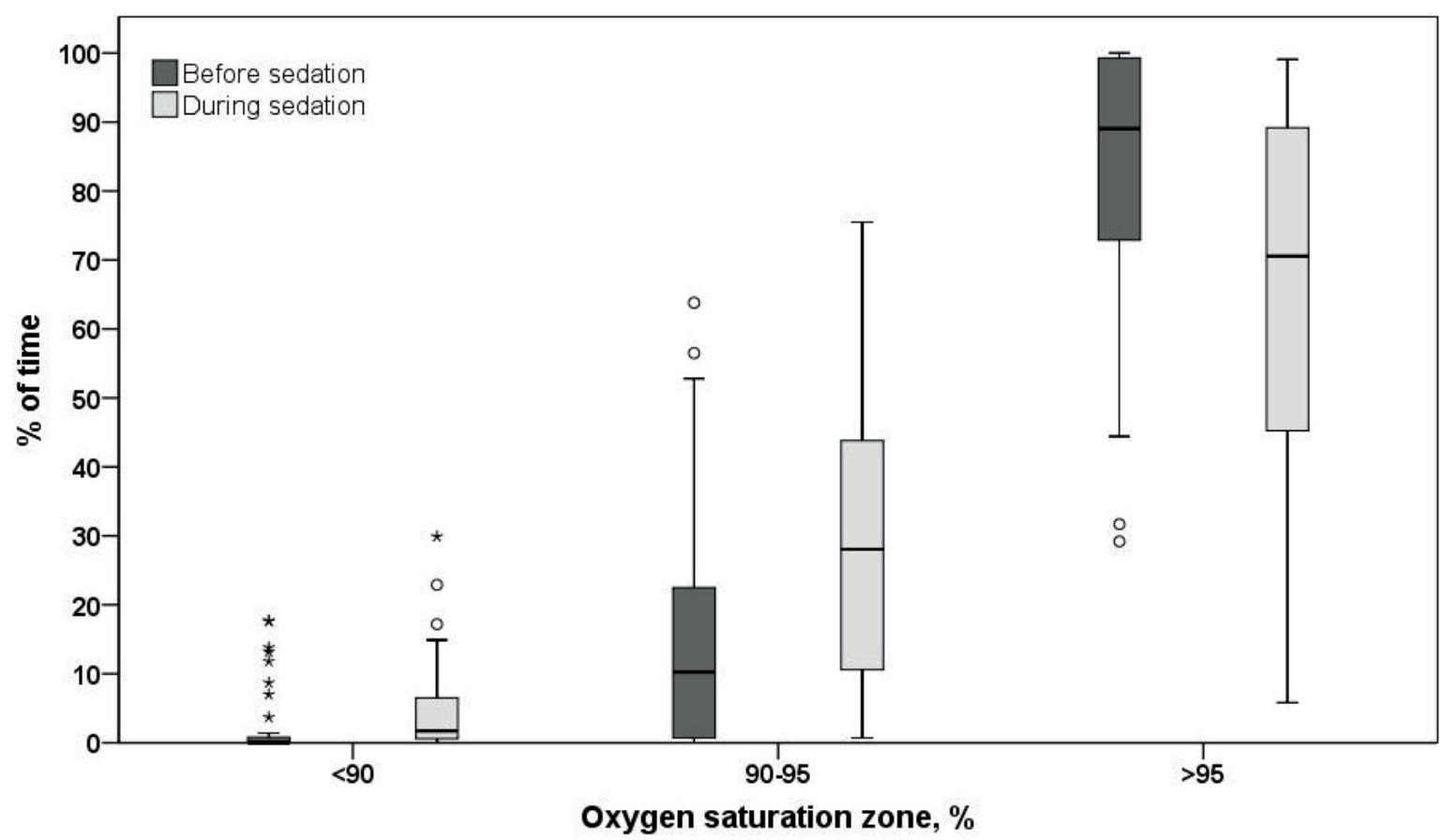

Fig. 1. Box plot of time spent with SpO2 within certain zones before sedation with chloral hydrate (dark gray) and during sedation with chloral hydrate (light gray) at a dose of $40 \mathrm{mg} / \mathrm{kg}$. The line across each box represents the median value. The bottom of the box represents the 25th percentile and the top of the box represents the 75th percentile. The lower and upper bars that extend from each box (whiskers) extend to 1.5 times the height of the box or to the minimum or maximum value. The closed circles indicate outliers and the asterisks are extreme outliers (values more than three times the height of the boxes).

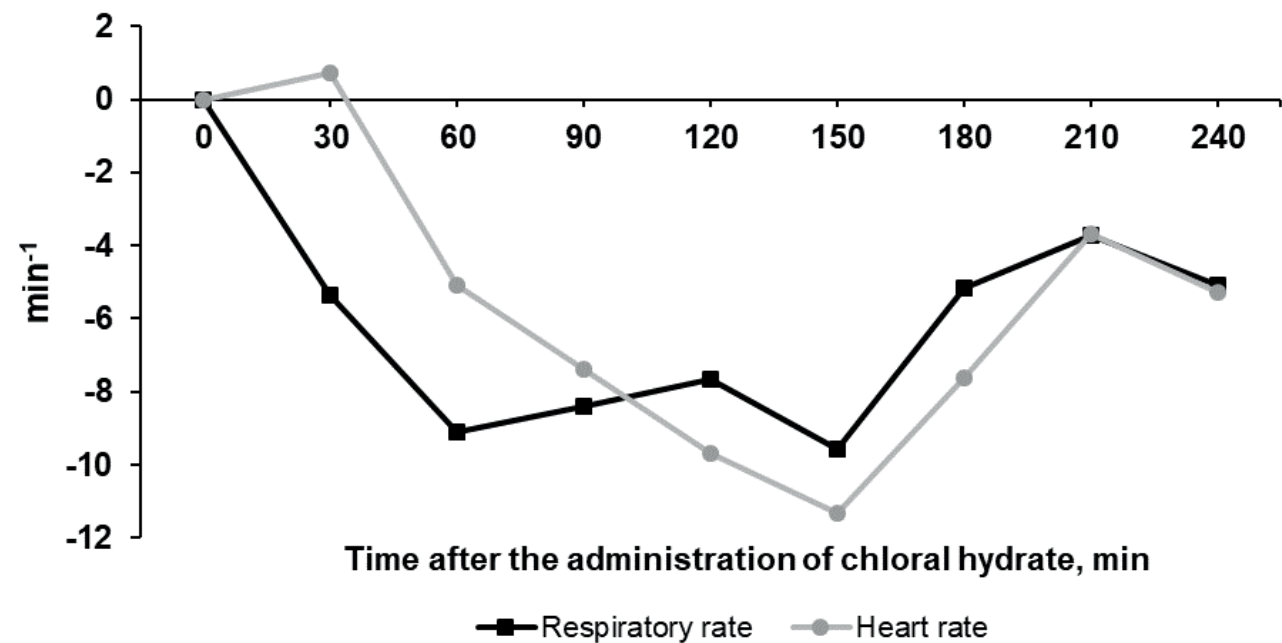

Fig. 2. Time course of the mean changes in the respiratory rate (black line) and heart rate (gray line) during sedation with chloral hydrate at a dose of $40 \mathrm{mg} / \mathrm{kg}$ relative to the mean baseline values before sedation (starting point is marked as a zero value). 


\section{Discussion}

Our study confirmed the impact of chloral hydrate at a dose of $40 \mathrm{mg} / \mathrm{kg}$ on cardiorespiratory parameters in newborns. All primary and secondary outcomes changed significantly after the administration of chloral hydrate. Nevertheless, the cardiorespiratory parameters remained within the normal ranges in the majority of newborns. For instance, one of our primary outcomes - mean basal $\mathrm{SpO}_{2}-$ decreased by $2 \%$, but the mean value after the administration of chloral hydrate remained above $95 \%$. At the same time, there was a small but important number of patients in whom we measured changes in cardiorespiratory parameters that differed considerably from the accepted normal ranges.

We also investigated the impact of chloral hydrate on the noninvasively measured $\mathrm{ETCO}_{2^{\prime}}$ which has been shown to be an accurate measure of ventilation in nonintubated newborns with normal respiratory function. ${ }^{18-20}$ Changes of $\mathrm{ETCO}_{2}$ during sedation with chloral hydrate have previously only been investigated in two retrospective studies, both of which included older children.,10 The increase in $\mathrm{ETCO}_{2}$ to an average of $30 \mathrm{mmHg}$ and to a maximum of $38 \mathrm{mmHg}$ with a decrease of respiratory rate from 61 to 51 breaths per minute in our study may indicate bradypneic hypoventilation. ${ }^{18,19,26}$ However, it is important to note that we measured only basal $\mathrm{ETCO}_{2}$ and that our results do not provide information on the percentages of time during which $\mathrm{ETCO}_{2}$ was above the expected levels. The equipment we used also does not allow quantitative measurement of tidal voulmes. We therefore cannot conclude whether our patients had hypopneic hypoventilation or not. ${ }^{26,27} \mathrm{~A}>20 \%$ increase in basal $\mathrm{ETCO}_{2}$ was found in 9 of 27 of our studied newborns (33\%). In contrast, in their retrospective study, Heistein et al. ${ }^{3}$ reported a $>20 \%$ increase from the baseline $\mathrm{ETCO}_{2}$ measurement after the administration of chloral hydrate in only 40 of 603 patients (6.6\%). This discrepancy could be attributed to the differences in age - the age of the patients in the study by Heistein et al. ${ }^{3}$ was between 1 month and 3 years, whereas our study included only newborns.

We also observed significantly higher percentages of time when $\mathrm{SpO}_{2}$ was $<95 \%$ after the administration of chloral hydrate in comparison with pre-sedation values. At the same time, the percentages of time when $\mathrm{SpO}_{2}$ was $<90 \%$ remained very low in most newborns both before and during sedation with chloral hydrate. There are currently no reference data on the normal percentages of time spent in each saturation zone for healthy full-term infants. ${ }^{23}$ The cut-off level of $95 \%$ was chosen because this was reported as the 5 th percentile of basal $\mathrm{SpO}_{2}$ for healthy term newborns. ${ }^{24}$

Our study also showed a significant increase in the estimated apnea-hypopnea index during sedation with chloral hydrate. The mean apnea-hypopnea index in the period after the administration of chloral hydrate was 3.4 events per hour. However, apneas of short duration are common in healthy term newborns, and are mostly central. ${ }^{23,28}$ The median central apnea index for 1-month-old newborns was reported to be 5-10 events per hour and the 95 th percentile was $45 .{ }^{23}$ Thus, given the fact that most of the recorded apneas were central in origin, the apnea-hypopnea index remained within normal limits even during sedation with chloral hydrate. It should be emphasized, however, that we used a nasal thermistor instead of a nasal pressure transducer to detect hypopneas, hence it is possible that we underestimated the number of hypopneas. ${ }^{29}$ Furthermore, since we did not objectively determine the total sleep time by analyzing neurophysiological variables, our calculation of the apnea-hypopnea index was only an approximation based on the number of apneas and hypopneas per hour of estimated sleep time.

During the course of sedation, the respiratory and heart rates decreased in most patients. The mean maximum decrease in the respiratory rate from 61 to 51 breaths per minute and the mean decrease in the heart rate from 138 to 127 beats per minute do not represent a deviation from the 
reported normal ranges. ${ }^{21,22}$ It is less plausible that the decrease in respiratory and heart rates was secondary to hypoxia or hypercapnia since both changes were clinically non-significant and because the mean $\mathrm{SpO}_{2}$ and $\mathrm{ETCO}_{2}$ remained within normal limits. However, the effects of chloral hydrate varied considerably among the newborns. While the respiratory and heart rates actually increased in some of the studied newborns, one newborn experienced a clinically important decrease in the respiratory rate and three participants experienced a clinically important decrease in heart rate. These findings are consistent with the report by Treluyer et al. ${ }^{8}$, who observed a decrease in the respiratory rate outside the normal limits for age in three of 19 children aged $2.13 \pm 1.43$ years. Our results are also compatible with the study by Heistein et al. ${ }^{3}$, where alterations in heart rate beyond the published normal ranges occurred in a minority $(1.4 \%)$ of the children between one month and three years of age, sedated with chloral hydrate.

In our study, the impact of chloral hydrate on the respiratory and heart rates was greatest 150 minutes after administration of chloral hydrate. Our results are not in agreement with the previously reported time-course of chloral hydrate sedation in patients aged between six months and six years, where the maximum clinical sedative effect was observed after 30 minutes, following which it gradually decreased. ${ }^{8}$ In another study in patients aged between three months and twelve years, the effects of sedation completely disappeared within the span of about 60 minutes..$^{5}$ This disagreement could be attributed to the prolonged terminal serum elimination half-life of chloral hydrate and its active metabolite in newborns, compared to the older population. ${ }^{6,15}$ Our findings imply that newborns should be monitored for at least three hours after sedation with chloral hydrate. Further studies should explore the temporal association between the clinically apparent effects of sedation and the measured effects of chloral hydrate on cardiorespiratory parameters.
It should also be emphasized that there was high inter-individual variability in our study, as evident from the large statistical dispersion of the measured variables. Furthermore, the variability of some of the cardiorespiratory parameters (e.g. time with $\mathrm{SpO}_{2}<95 \%$, time with $\mathrm{SpO}_{2}<90 \%$ and the apnea-hypopnea index) was markedly greater after the administration of chloral hydrate than before sedation. This clearly shows that chloral hydrate does not have the same effects on all newborns. As a consequence, we recorded values outside the normal limits only in individual newborns, while the average values remained within the normal range.

This is one of the few existing studies focusing on the effects of chloral hydrate sedation in the most vulnerable pediatric age group. We did not rely solely on monitoring the clinically discernible adverse events of sedation. Instead, we measured the impact of chloral hydrate on cardiorespiratory parameters in a prospective manner, which is a significant advantage of our study. To our knowledge, this is the first study that investigated the effects of chloral hydrate using polygraphy. In previous studies, vital signs were recorded at fixed intervals (every 5 minutes $^{3,4}$ or every 15-30 minutes ${ }^{8,9}$ ), whereas in our case, we used computer processing to analyze continuously measured parameters. Consequently, our findings do not support the results of a prior observational study by Treluyer et al. $^{8}$ in 20 children aged $2.13 \pm 1.43$ years, in whom no statistically significant change in the vital functions was found after rectal administration of $75 \mathrm{mg} / \mathrm{kg}$ of chloral hydrate. Similar results were found in the study by Coskun et al. ${ }^{30}$ in 360 patients aged $19 \pm 4.5$ months.

The major limitation of the present study is the difference in the duration of polygraphic recordings before and after the administration of chloral hydrate. For a more accurate comparison of these two periods, the recording time should be the same length for both parts of the study. Furthermore, the two parts of the study are not 
entirely comparable due to the unequal external conditions during the recording and thus possible confounding factors. Future studies are therefore needed, together with a superior study protocol involving cardiorespiratory recording on two separate, consecutive days, of the same duration and at the same time of the day in each case, on the first day without sedation and on the second day after the administration of chloral hydrate. To directly distinguish between the effects of chloral hydrate and the influences caused by varying sleep stages, a full-channel polysomnography, rather than polygraphy, would be the more appropriate investigation method.

The small sample size is another limitation of our research, especially for the subgroup in which we measured $\mathrm{ETCO}_{2}$. In addition, our study population was not homogeneous. Participating newborns differed in gestational age, antenatal history, Apgar score and clinical diagnoses. At the same time, great interindividual variability could very well be the advantage of our research, since this sample is representative - it reflects the actual population in which we use chloral hydrate sedation in clinical practice.

To conclude, in a group of term newborns with normal cardiovascular and respiratory physiology, sedation with oral chloral hydrate at a dose of $40 \mathrm{mg} / \mathrm{kg}$ caused mild changes in cardiorespiratory parameters. Oxygenation and ventilation remained adequate in most cases, but the cardiorespiratory effects of chloral hydrate varied considerably among newborns. Our results could serve as a pilot for a larger study to determine the safety of chloral hydrate sedation in newborns.

\section{REFERENCES}

1. Avlonitou E, Balatsouras DG, Margaritis E, Giannakopoulos P, Douniadakis D, Tsakanikos M. Use of chloral hydrate as a sedative for auditory brainstem response testing in a pediatric population. Int J Pediatr Otorhinolaryngol 2011; 75: 760-763.
2. Allegaert K, Daniels H, Naulaers G, Tibboel D, Devlieger $\mathrm{H}$. Pharmacodynamics of chloral hydrate in former preterm infants. Eur J Pediatr 2005; 164: 403-407.

3. Heistein LC, Ramaciotti C, Scott WA, Coursey M, Sheeran PW, Lemler MS. Chloral hydrate sedation for pediatric echocardiography: physiologic responses, adverse events, and risk factors. Pediatrics 2006; 117: e434-e441.

4. Finnemore A, Toulmin H, Merchant N, et al. Chloral hydrate sedation for magnetic resonance imaging in newborn infants. Paediatr Anaesth 2014; 24: 190-195.

5. Marchi A, Orrù A, Manai ME, Chelo C, Lettieri B, Corbucci GG. Deep sedation for magnetic resonance imaging. Personal experience. Minerva Anestesiol 2004; 70: 53-61.

6. Jacqz-Aigrain E, Burtin P. Clinical pharmacokinetics of sedatives in neonates. Clin Pharmacokinet 1996; 31: 423-443.

7. Sezer T, Alehan F. Chloral hydrate versus hydroxyzine $\mathrm{HCl}$ for sedation prior to pediatric sleep EEG recording. Int J Neurosci 2013; 123: 719723.

8. Treluyer JM, Andre C, Carp PF, et al. Sedation in children undergoing CT scan or MRI: effect of time-course and tolerance of rectal chloral hydrate. Fundam Clin Pharmacol 2004; 18: 347-350.

9. Sanatkar M, Ghassemi F, Khodabande A, et al. Use of chloral hydrate as a sedative agent before general anesthesia in pediatric population undergoing eye examination. Arch Anesthesiol Crit Care 2015; 1: 8083.

10. Cortellazzi P, Lamperti M, Minati L, Falcone C, Pantaleoni C, Caldiroli D. Sedation of neurologically impaired children undergoing MRI: a sequential approach. Paediatr Anaesth 2007; 17: 630-636.

11. Nordt SP, Rangan C, Hardmaslani M, Clark RF, Wendler C, Valente M. Pediatric chloral hydrate poisonings and death following outpatient procedural sedation. J Med Toxicol 2014; 10: 219-222.

12. Coté CJ, Wilson S. Guidelines for monitoring and management of pediatric patients during and after sedation for diagnostic and therapeutic procedures: an update. Pediatrics 2006; 118: 2587-2602.

13. Mace SE, Brown LA, Francis L, et al. Clinical policy: critical issues in the sedation of pediatric patients in the emergency department. Ann Emerg Med 2008; 51: 378-399. e1-e57.

14. Reynolds J, Rogers A, Capehart S, Manyang P, Watcha MF. Retrospective comparison of intranasal dexmedetomidine and oral chloral hydrate for sedated auditory brainstem response exams. Hosp Pediatr 2016; 6: 166-171. 
15. Mayers DJ, Hindmarsh KW, Sankaran K, Gorecki DK, Kasian GF. Chloral hydrate disposition following single-dose administration to critically ill neonates and children. Dev Pharmacol Ther 1991; 16: 71-77.

16. Litman RS, Soin K, Salam A. Chloral hydrate sedation in term and preterm infants: an analysis of efficacy and complications. Anesth Analg 2010; 110: 739-746.

17. Malviya S, Voepel-Lewis T, Tait AR. Adverse events and risk factors associated with the sedation of children by nonanesthesiologists. Anesth Analg 1997; 85: 1207-1213.

18. Coates BM, Chaize R, Goodman DM, Rozenfeld RA. Performance of capnometry in non-intubated infants in the pediatric intensive care unit. BMC Pediatr 2014; 14: 163.

19. Tai CC, Lu FL, Chen PC, et al. Noninvasive capnometry for end-tidal carbon dioxide monitoring via nasal cannula in nonintubated neonates. Pediatr Neonatol 2010; 51: 330-335.

20. Hagerty JJ, Kleinman ME, Zurakowski D, Lyons AC, Krauss B. Accuracy of a new low-flow sidestream capnography technology in newborns: a pilot study. J Perinatol 2002; 22: 219-225.

21. O'Leary F, Hayen A, Lockie F, Peat J. Defining normal ranges and centiles for heart and respiratory rates in infants and children: a cross-sectional study of patients attending an Australian tertiary hospital paediatric emergency department. Arch Dis Child 2015; 100: 733-737.
22. Fleming S, Thompson M, Stevens R, et al. Normal ranges of heart rate and respiratory rate in children from birth to 18 years of age: a systematic review of observational studies. Lancet 2011; 377: 1011-1018.

23. $\mathrm{Ng} \mathrm{DK}, \mathrm{Chan} \mathrm{CH}$. A review of normal values of infant sleep polysomnography. Pediatr Neonatol 2013; 54: 82-87.

24. Terrill PI, Dakin C, Hughes I, Yuill M, Parsley C. Nocturnal oxygen saturation profiles of healthy term infants. Arch Dis Child 2015; 100: 18-23.

25. Shah PS, Hakak H, Mohamed A, Shah J, Young J, Kelly E. Oxygen saturation profile in late-preterm and term infants: a prospective cohort study. J Perinatol 2014; 34: 917-920.

26. Krauss B, Hess DR. Capnography for procedural sedation and analgesia in the emergency department. Ann Emerg Med 2007; 50: 172-181.

27. Berry RB, Budhiraja R, Gottlieb DJ, et al. Rules for scoring respiratory events in sleep: update of the 2007 AASM Manual for the Scoring of Sleep and Associated Events. Deliberations of the Sleep Apnea Definitions Task Force of the American Academy of Sleep Medicine. J Clin Sleep Med 2012; 8: 597-619.

28. Gaultier C. Review article: Sleep apnea in infants. Sleep Med Rev 1999; 3: 303-312.

29. Farré R, Montserrat JM, Rotger M, Ballester E, Navajas D. Accuracy of thermistors and thermocouples as flow-measuring devices for detecting hypopnoeas. Eur Respir J 1998; 11: 179-182.

30. Coskun S, Yuksel H, Onag A. Chloralhydrate in children undergoing echocardiography. Indian J Pediatr 2001; 68: 319-322. 